\title{
EPR determination of three-dimensional correlations below the ferroelectric phase transition in pseudo-one-dimensional $\mathrm{CsH}_{2} \mathrm{PO}_{4}: \mathrm{Cu}^{2+}$
}

\author{
S. Waplak, ${ }^{*}$ V. Hugo Schmidt, and John E. Drumheller \\ Department of Physics, Montana State University, Bozeman, Montana 59717
}

(Received 26 June 1986)

\begin{abstract}
The polarization fluctuations of ferroelectric $\mathrm{CsH}_{2} \mathrm{PO}_{4}$ above $T_{c}$ are generally classified as a quasi-one-dimensional phenomenon. The temperature dependence of the EPR line splitting of the $S=1$ state of the $\mathrm{Cu}^{2+}$ tetramer, however, exhibits a critical exponent below $T_{c}$ of $\beta=0.5$ indicating that the correlations associated with the order parameter are three dimensional below $T_{c}$.
\end{abstract}

The phase transition in monoclinic $\mathrm{CsH}_{2} \mathrm{PO}_{4}$ (CDP) exhibits a number of unusual properties which are characteristic of one-dimensional systems. ${ }^{1-4}$ The ferroelectricity in CDP is associated with the ordering of hydrogen between two equilibrium sites in the short $\mathrm{O}-\mathrm{H} \cdots \mathrm{O}$ bonds linking the $\mathrm{PO}_{4}$ groups into zigzag chains parallel to the $b$ axis. These chains are linked into two-dimensional (2D) layers by ordered hydrogen bonds lying roughly along the $c$ axis and cesium atoms lying between these hydrogenbonded planes. ${ }^{5}$ There are no hydrogen bonds along the $a$ axis and the crystal has a cleavage plane perpendicular to the $a$ axis. Neutron scattering studies ${ }^{6}$ show that significant short-range order develops along the chains at a temperature well above $T_{c}$. The weaker interchain coupling responsible for the ferroelectric transition causes virtually no correlation between the layers until one gets quite close to $T_{c}$. The dielectric constant in CDP shows a crossover ${ }^{4}$ between a region $3 \mathrm{~K}<T-T_{c} \leq 90 \mathrm{~K}$ dominated by $1 \mathrm{D}$ correlations and a region $0 \mathrm{~K}<T-T_{c} \leq 3 \mathrm{~K}$ dominated by $3 \mathrm{D}$ correlations. The $1 \mathrm{D}$ correlation has been confirmed by ${ }^{31} \mathrm{P}$ and deuteron resonance studies for partially deuterated CDP. ${ }^{4}$ An apparent deviation from the quasione-dimensional model has been found, however, in the region of $0 \mathrm{~K}<T-T_{c} \leq 20 \mathrm{~K}$ by Deguchi, Okau, and Nakamura. ${ }^{7}$ The aim of this work is to examine the temperature dependence of the order parameter of the phase transition by means of an EPR study of the line splitting for the fine-structure component of the $\mathrm{Cu}^{2+}$ tetramer spectrum in CDP.

$\mathrm{CsH}_{2} \mathrm{PO}_{4}$ doped with $0.3 \mathrm{~mol} \% \mathrm{CuCl}_{2} \cdot 2 \mathrm{H}_{2} \mathrm{O}$ shows the usual $\mathrm{Cu}^{2+} S=\frac{1}{2}$ single-ion spectrum at room temperature. Below about $250 \mathrm{~K}$ the fine-structure spectra were observed and described by $\mathrm{us}^{8}$ as arising from the $\mathrm{Cu}^{2+}$ tetramers with an effective spin $S=2$ and spin-Hamiltonian parameters: $g_{\|}=2.2575, g_{\perp}=2.1866, \quad D=0.181$ $\mathrm{cm}^{-1}, E=-0.081 \mathrm{~cm}^{-1}, a=0.001 \mathrm{~cm}^{-1}, b=0$, and $c=-0.021 \mathrm{~cm}^{-1}$. Also the $0 \rightarrow-1$ transition within the $S=1$ multiplet of the tetramer was reported to have approximate ${ }^{8}$ parameters $D_{x}=0.543$ and $E_{x}=-0.159 \mathrm{~cm}^{-1}$ obtained from a phenomenological theory of the tetrameric clusters. The superexchange interaction between pairs of $\mathrm{Cu}^{2+}(S=1)$ complexes arises through $\mathrm{Cu}-\mathrm{O}-\mathrm{P}-\mathrm{O}-\mathrm{Cu}$ paths. Both pairs are coupled by the hydrogen bonds along the $c$ axis. The tetramer model is shown in Fig. 1 . The excess charge compensation for the $\mathrm{Cu}^{2+}$ ion which replaces the $\mathrm{Cs}^{+}$ion is presumably provided by a proton vacancy. Figure 2 shows the EPR line splitting for the $0 \rightarrow-1$ transition for the $S=1$ multiplet versus temperature.

The EPR line position above $T_{c}$ for the $0 \rightarrow-1$ transition is given by the expression ${ }^{8}$

$$
\begin{aligned}
H_{0}=\left(1 / g \mu_{\beta}\right)\left\{h v+\left(\frac{1}{2}\right)\right. & {\left[D_{x}\left(3 \cos ^{2} \theta-1\right)\right.} \\
+ & \left.\left.3 E_{x} \sin ^{2} \theta \cos (2 \phi)\right]\right\},
\end{aligned}
$$

where $\theta$ and $\phi$ are the polar and azimuthal angles of the external magnetic field $H_{0}$ with respect to the $x, y, z$ crystal-field axes. The values of $D_{x}$ and $E_{x}$ for $T \geq T_{c}$ obtained by fitting the EPR line anisotropy for the triplet spectrum are 0.462 and $-0.105 \mathrm{~cm}^{-1}$, respectively. The parameters $D_{x}, E_{x}$, and $\theta$ are not strongly temperature dependent below $T_{c}$, and the $\phi$ dependence below $T_{c}$ is negligible since $\Delta \phi$ is less than $2^{\circ}$. Therefore, the equation for the line splitting below $T_{c}$ can be approximated from (1) to give

$$
g \mu_{\beta} \Delta H=3 \Delta E \sin ^{2} \theta \cos (2 \phi),
$$

where $\Delta E$ arises because of the phase transition. For the external magnetic field orientation $\theta=\phi=20^{\circ}, \Delta H$ is

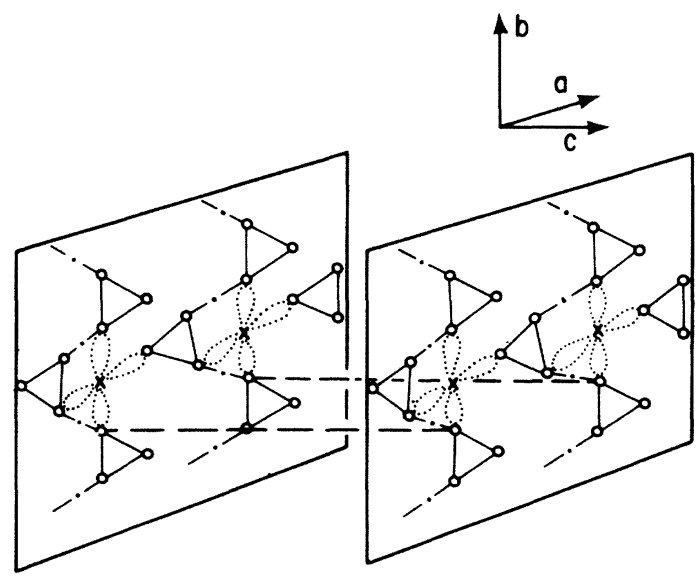

FIG. 1. Model of the tetrameric copper complex in CDP showing the superexchange paths. Two $a-b$ planes are shown, joined along the $c$ axis by ordered $\mathrm{H}$ bonds represented by a dashed line (--). Some of the disordered $H$ bonds are represented by a dashed-dotted line (....). 


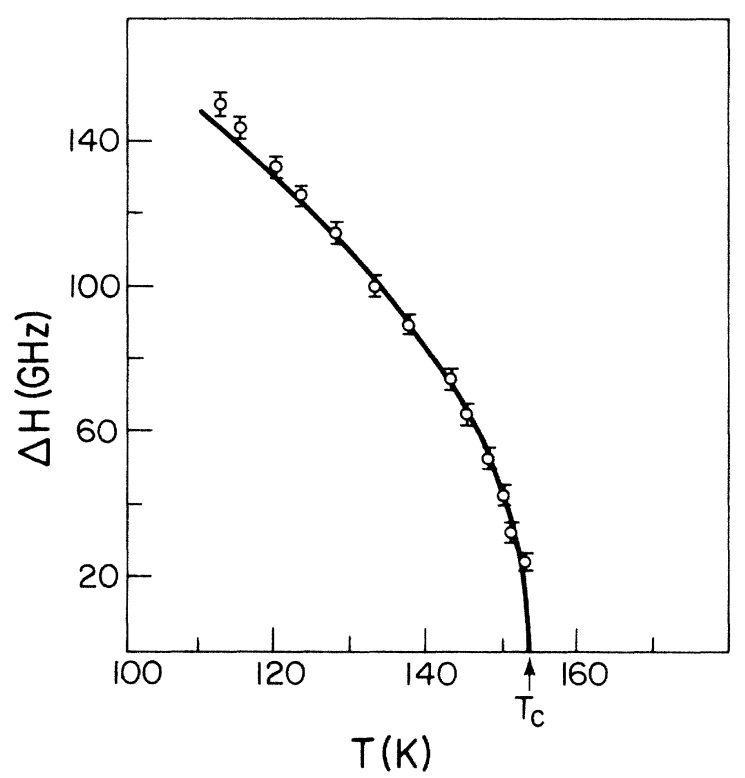

FIG. 2. EPR line splitting for $0 \rightarrow-1$ transition for $S=1$ multiplet vs temperature. Circles represent experimental data and the solid line is the fit of Eq. 4 with $\beta=0.5$.

given by

$$
\Delta H(\mathrm{GHz})=2.64 \times 10^{3} \Delta E\left(\mathrm{~cm}^{-1}\right),
$$

with

$$
\Delta E=E_{x}\left[\left(T_{c}-T\right) / T_{c}\right]^{\beta} .
$$

According to Itoh, Hagiwara, and Nakamura, ${ }^{9}$ just below $T_{c}$ the ordering of hydrogen $\mathrm{H}(2)$ to either of the two possible sites results in a slight change of $\mathrm{PO}_{4}$ tetrahedron orientation consisting of rotation around the $\mathrm{P}-\mathrm{O}(2)$ bond and a shift of the $\mathrm{P}$ atom position along the $b$ axis. These displacements are responsible for the polari- zation. The spin-Hamiltonian parameter $\Delta E$ should reflect these displacements and describe the temperature evolution of the phase transition order parameter.

In the temperature region $0 \mathrm{~K}<T_{c}-T \leq 30 \mathrm{~K}$, the $\Delta H_{0}$ value is satisfactorily described by Eqs. (3) and (4) with $\beta=0.48 \pm 0.02$ as shown in Fig. 2. This agrees within experimental error with the mean-field value of 0.5 for the critical exponent $\beta$, as expected for 3D correlations of the displacements leading to spontaneous polarization. This critical behavior of the EPR line splitting below $T_{c}$ shows that the $\mathrm{PO}_{4}$ distortion is governed by $3 \mathrm{D}$ correlations. Such $3 \mathrm{D}$ ordering of the $\mathrm{PO}_{4}$ tetrahedra had been suggested previously by Topic et al. ${ }^{10}$ from their deuteron NMR studies in $\mathrm{CsD}_{2} \mathrm{PO}_{4}$.

Results from other authors for $\beta$ determined from other types of measurements are not in complete agreement. Our analysis of the spontaneous polarization $\left(P_{s}\right)$ data of Yasuda et al. ${ }^{11}$ for $\mathrm{CsH}_{2} \mathrm{PO}_{4}$ at 1 bar pressure yields $\beta=0.50 \pm 0.04$ for the points within $5 \mathrm{~K}$ of $T_{c}$. Levstik et al. ${ }^{12}$ found a linear relation between $P_{s}^{2}$ and $T$ for $\mathrm{CsD}_{2} \mathrm{PO}_{4}$, indicating an exponent $\beta=0.5$. However, Blinc et al. ${ }^{4}$ found an exponent $\beta=0.35 \pm 0.05$ for the deuterated crystal, $\mathrm{CsD}_{2} \mathrm{PO}_{4}$, from both ${ }^{31} \mathrm{P}$ NMR chemical shift and deuteron NMR measurements, and stated that this agreed with spontaneous polarization results for both $\mathrm{CsH}_{2} \mathrm{PO}_{4}$ and $\mathrm{CsD}_{2} \mathrm{PO}_{4}$ without giving a reference for the spontaneous polarization measurements.

In conclusion, our EPR results indicate 3D correlations of atomic displacements in CDP below $T_{c}$, in contrast to the $1 \mathrm{D}$ correlations observed above (except very near to) $T_{c}$. Discrepancies exist in the critical exponent $\beta$ reported for different experiments performed below $T_{c}$. However, a $\beta$ less than 0.5 could be attributed to nearness to a tricritical point (for which the mean-field $\beta$ is 0.25 ) rather than to lower-dimensional effects.

This work was supported by National Science Foundation Grants No. DMR-8205280 and No. DMR-8403993.
*Permanent address: Institute of Molecular Physics, Polish Academy of Sciences, Poznán, Poland.

'Y. Uesu and J. Kobayashi, Phys. Status Solidi A 34, 475 (1976).

${ }^{2}$ H. Matsunaga, K. Itoh, and E. Nakamura, J. Phys. Soc. Jpn. 48, 2011 (1980).

${ }^{3}$ E. Kanda and T. Fujimura, J. Phys. Soc. Jpn. 43, 1813 (1977).

${ }^{4}$ R. Blinc, B. Žekš, A. Levstik, C. Filipič, J. Slak, M. Burgar, I. Zupančič, L. A. Shuvalov, and I. Baranov, Phys. Rev. Lett. 43, 231 (1979).

${ }^{5}$ Y. Iwata, N. Koyano, and I. Shibuya, J. Phys. Soc. Jpn. 49, 304 (1980).

${ }^{6}$ B. C. Frazer, D. Semmingsen, W. D. Ellenson, and G. Shirane,
Phys. Rev. B 20, 2745 (1979).

${ }^{7}$ K. Deguchi, E. Okaue, and E. Nakamura, J. Phys. Soc. Jpn. 51, 3569 (1982).

${ }^{8}$ S. Waplak, V. H. Schmidt, and J. E. Drumheller, Phys. Rev. B 32, 48 (1985).

${ }^{9}$ K. Itoh, T. Hagiwara, and E. Nakamura, J. Phys. Soc. Jpn. 52, 2626 (1983).

${ }^{10}$ B. Topič, V. Rutar, J. Slak, M. I. Burgar, S. Žumer, and R. Blinc, Phys. Rev. B 21, 2695 (1980).

${ }^{11}$ N. Yasuda, S. Fujimoto, M. Okamoto, H. Shimizu, K. Yoshino, and Y. Inuishi, Phys. Rev. B 20, 2755 (1979).

${ }^{12}$ A. Levstik, R. Blinc, P. Kadaba, S. Čižikov, I. Levstik, and C. Filipič, Solid State Commun. 16, 1339 (1975). 